\title{
Solving the Dym initial value problem in reproducing kernel space
}

\author{
P. Bakhtiari ${ }^{a}$, S. Abbasbandy ${ }^{a *}$, and R. A. Van Gorder ${ }^{b}$ \\ ${ }^{a}$ Department of Mathematics, Imam Khomeini International University, Qazvin, 34149-16818, Iran \\ ${ }^{b}$ Mathematical Institute, University of Oxford, Oxford, OX2 6GG, United Kingdom \\ *Corresponding author: abbasbandy@yahoo.com, Tel.:+98(912)1305326.
}

\begin{abstract}
We consider two numerical solution approaches for the Dym initial value problem using the reproducing kernel Hilbert space method. For each solution approach, the solution is represented in the form of a series contained in the reproducing kernel space, and a truncated approximate solution is obtained. This approximation converges to the exact solution of the Dym problem when a sufficient number of terms are included. In the first approach, we avoid having to perform the Gram-Schmidt orthogonalization process on the basis functions, and this will decrease the computational time. Meanwhile, in the second approach, working with orthonormal basis elements gives some numerical advantages, despite the increased computational time. The latter approach also permits a more straightforward convergence analysis. Therefore, there are benefits to both approaches. After developing the reproducing kernel Hilbert space method for the numerical solution of the Dym equation, we present several numerical experiments in order to show that the method is efficient, and can obtain accurate approximations to the Dym initial value problem for sufficiently regular initial data after relatively few iterations. We present the absolute error of the results when exact solutions are known, and residual errors for other cases. The results suggest that numerically solving the Dym initial value problem in reproducing kernel space is a useful approach for obtaining accurate solutions in an efficient manner.
\end{abstract}

Keywords: Dym equation; Reproducing kernel method; initial value problem; convergence analysis

\section{Introduction}

The Dym equation is an important nonlinear partial differential equation which is integrable and finds applications in several physical systems. The Harry Dym equation was discovered by H. Dym in 1973-1974; its first appearance in the literature occurred in a 1975 paper of Kruskal [15]. The equation is one example of an integrable system and it has been related to the $\mathrm{KdV}$ equation through use of a Liouville transformation of the relevant Sturm-Liouville operator for this equation [12]. This demonstrates the model's applicability to the study of the fluid dynamics of water waves. Additionally, the equation arises in the study of the Saffman-Taylor problem related to surface tension [14]. The Dym equation in two space variables has also been linked to the study of the integrable KP equation [23]. This matter has been discussed in $[9,19,13,22]$ thoroughly and one can consult them.

The Dym equation is written as

$$
u_{t}=u^{3} u_{x x x},
$$

on the domain $\mathbb{R} \times[0, \infty)$. In this paper, we seek a method of solution for the Dym initial value problem that permits fairly arbitrary (although sufficiently regular) initial conditions

$$
u(x, 0)=f(x) .
$$

One exact solution of the Dym equation is $u(x, t)=\left(-3 c\left[x+c^{2} t\right]\right)^{2 / 3}$, as show in [20]. In general, exact solutions are not be expected for general initial data $f(x)$. Due to the nonlinearity of the equation, in particular the fact that the highest space derivative is multiplied by a power of the function, numerical solutions can be hard to obtain.

The aim of this paper is to introduce a numerical technique based on reproducing kernel Hilbert space methods in order to solve the Dym problem. The concept of reproducing kernel introduced by Zaremba in 1908 [26] in harmonic functions with boundary conditions, however, it was not formally studied until Bergman attempted in providing a 
fundamental framework for its theory during the forth decade in the 20th century [4, 5]. In 1950, Aronszajn produced a systematic reproducing kernel space method based on the Bergman's works [2,3]. Reproducing kernel theory has important applications in numerical analysis. Recently, a lot of research works have been devoted to the developments and applications of reproducing kernel methods. These methods have successfully been applied to several nonlinear problems, such as nonlinear system of boundary value problems, nonlinear initial value problems, singular nonlinear two-point periodic boundary value problems and singularly perturbed turning point problems $[1,6,7,8,16,17,18,10$, $11,24,25]$. This method has ability to solve different problems effectively and has relatively simple implementation.

The remainder of the paper is organized as follows. In Section 2, we give some preliminaries for the reproducing kernel space. In Section 3, we introduce both numerical methods, the first for non-orthogonal basis functions, and the second using orthogonal basis functions. In Section 4, we provide a convergence analysis of for approximate solution obtained via the second method. In Section 5, we provide several numerical experiments, in order to show that the numerical approach is both accurate and efficient. Finally, concluding remarks are provided in Section 6.

\section{Reproducing kernel method preliminaries}

We want to utilize an appropriate reproducing kernel space for solving Eq. (1) under the given initial conditions $u(x, 0)=f(x)$. In this section, we discuss needed preliminary results before constructing such a numerical method.

In order to use the reproducing kernel space to solve (1), we need to first homogenize it. To begin, we define $u(x, t)=\vartheta(x, t)+f(x)$, and consequently we can rewrite (1) as follows

$$
\begin{gathered}
\vartheta_{t}=[\vartheta(x, t)+f(x)]^{3}\left[\frac{\partial^{3}}{\partial x^{3}} \vartheta(x, t)+f^{\prime \prime \prime}(x)\right], \\
\vartheta(x, 0)=0 .
\end{gathered}
$$

Note also that we shall consider the problem domain consisting of a bounded spatial part, $\Phi=[0, X] \times[0, \infty)$. Under an appropriate non-dimensionalization, $x=X \hat{x}$ and $t=X^{3} \hat{t}$, we may scale the space and time variables so that the domain $\Phi=[0,1] \times[0, \infty)$ may always be considered. In the following analysis, we shall drop the hats.

We present some necessary definitions and theorems in the theory of reproducing kernel spaces.

Consider the following reproducing kernel space ${ }^{0} \omega_{2}^{2}([0, \infty))$, which is defined as

$$
\begin{aligned}
& { }^{0} \omega_{2}^{2}([0, \infty))=\left\{v(t) \mid v(t), v^{\prime}(t)\right. \text { are absolutely continuous functions, } \\
& \left.\qquad v, v^{\prime}(t), v^{\prime \prime}(t) \in L^{2}([0, \infty)), v(0)=0\right\} .
\end{aligned}
$$

The reproducing kernel space ${ }^{0} \omega_{2}^{2}([0, \infty))$ is endowed with the inner product, $\left\langle v_{1}, v_{2}\right\rangle_{0} \omega_{2}^{2}$, defined by

$$
\left\langle v_{1}, v_{2}\right\rangle_{\omega_{2}^{2}[0, \infty)}=\int_{0}^{\infty}\left\{4 v_{1}(t) v_{2}(t)+5 v_{1}^{\prime}(t) v_{2}^{\prime}(t)+v_{1}^{\prime \prime}(t) v_{2}^{\prime \prime}(t)\right\} \mathrm{d} t
$$

This defines the norm $\|v\|_{\omega_{2}^{2}}=\sqrt{\langle v, v\rangle_{\omega_{2}^{2}}}$.

Similarly, we define the reproducing kernel space $\omega_{2}^{4}([0,1])$ by

$$
\omega_{2}^{4}([0,1])=\left\{v(x) \mid v^{(3)}(x) \text { is an absolutely continuous, } v^{(4)}(x) \in L^{2}([0,1])\right\} .
$$

The inner product and norm for $\omega_{2}^{4}([0,1])$ are given, respectively, by

$$
\left\langle v_{1}, v_{2}\right\rangle_{\omega_{2}^{4}}=\sum_{i=0}^{3} v_{1}^{(i)}(0) v_{2}^{(i)}(0)+\int_{0}^{1} v_{1}^{(4)}(x) v_{2}^{(4)}(x) \mathrm{d} x, \quad\|v\|_{\omega_{2}^{4}}=\sqrt{\langle v, v\rangle_{\omega_{2}^{4}}} .
$$

The existence of the reproducing kernel of a Hilbert space is due to the Riesz Representation Theorem. It is known that the reproducing kernel is unique. Before any further discussion, we need to give the reproducing kernel of $\omega_{2}^{4}([0,1])$, and that of ${ }^{0} \omega_{2}^{2}([0, \infty))$. Their respective reproducing kernels are found to be

$$
R_{\zeta}(x)= \begin{cases}\frac{1}{5040}\left(-\zeta^{7}+35(\zeta+4) \zeta^{3} x^{3}-21\left(\zeta^{3}-60\right) \zeta^{2} x^{2}+7\left(\zeta^{5}+720\right) \zeta x+5040\right), & x \leq \zeta, \\ \frac{1}{5040}\left(-x^{7}+7 \zeta\left(x^{5}+720\right) x+35 \zeta^{3}(x+4) x^{3}-21 \zeta^{2}\left(x^{3}-60\right) x^{2}+5040\right), & x>\zeta\end{cases}
$$


and

$$
K_{\eta}(t)= \begin{cases}\frac{1}{12}\left(e^{-2 \eta-2 t}-\frac{1}{12} e^{2 t-2 \eta}-\frac{e^{-\eta-t}}{6}+\frac{e^{t-\eta}}{6}\right), & t \leq \eta, \\ \frac{1}{6}\left(\left(e^{\eta}-e^{-\eta}\right) e^{-t}-\frac{1}{12}\left(e^{4 \eta}-1\right) e^{-2 \eta-2 t}\right), & t>\eta,\end{cases}
$$

respectively.

We next define the binary function space ${ }^{0} \omega_{2}^{(4,2)}(\Phi)$ by

$$
\begin{aligned}
{ }^{0} \omega_{2}^{(4,2)}(\Phi) & =\omega_{2}^{4}([0,1]) \times{ }^{0} \omega_{2}^{2}([0, \infty)) \\
& =\left\{u(x, t) \mid \frac{\partial^{4} u(x, t)}{\partial x^{3} \partial t} \text { is completely continuous in } \Phi, \frac{\partial^{6} u(x, t)}{\partial x^{4} \partial t^{2}} \in L^{2}(\Phi), u(x, 0)=0\right\} .
\end{aligned}
$$

Before continuing on to the numerical method, we shall need two results.

Theorem 2.1. ${ }^{0} \omega_{2}^{(4,2)}(\Phi)=\omega_{2}^{4}([0,1]) \times{ }^{0} \omega_{2}^{2}([0, \infty))$ is a reproducing kernel space which has reproducing kernel

$$
\Omega_{(\zeta, \eta)}(x, t)=R_{\zeta}(x) \kappa_{\eta}(t),
$$

where $R_{\zeta}(x), \kappa_{\eta}(t)$ are the reproducing kernels of $\omega_{2}^{4}([0,1])$ and ${ }^{0} \omega_{2}^{2}([0, \infty))$, such that for any $u(x, t) \in{ }^{0} \omega_{2}^{(4,2)}(\Phi)$ we have

$$
u(\zeta, \eta)=\left\langle u(x, t), \Omega_{(\zeta, \eta)}(x, t)\right\rangle_{\omega_{\omega}^{(4,2)}} .
$$

Proof 2.1. See [6].

It is easy to define the reproducing kernel space $\omega_{2}^{(1,1)}(\Phi)$ such as ${ }^{0} \omega_{2}^{(4,2)}(\Phi)$. Therefore, we can certainly assume that $\Upsilon_{(\zeta, \eta)}(x, y)$ is the reproducing kernel space $\omega_{2}^{(1,1)}(\Phi)$. Moreover, the linear operator $\mathcal{L}:{ }^{0} \omega_{2}^{(4,2)}(\Phi) \longrightarrow{ }^{0} \omega_{2}^{(1,1)}(\Phi)$ is bounded and defined by

$$
(\mathcal{L} \vartheta)(x, t) \equiv \frac{\partial}{\partial t} \vartheta(x, t),
$$

where

$$
\mathcal{F}\left(x, t, \vartheta(x, t), \partial_{x x x} \vartheta(x, t)\right) \equiv[\vartheta(x, t)+f(x)]^{3}\left[\frac{\partial^{3}}{\partial x^{3}} \vartheta(x, t)+f^{\prime \prime \prime}(x)\right] .
$$

Hence, Eq. (3) is converted to

$$
\begin{gathered}
\mathcal{L} \vartheta(x, t)=\mathcal{F}\left(x, t, \vartheta(x, t), \partial_{x x x} \vartheta(x, t)\right), \\
\vartheta(x, 0)=0 .
\end{gathered}
$$

We then set $\varphi_{i}(x, t)=\Upsilon_{\left(x_{i}, t_{i}\right)}(x, t)$, in which $\left\{\left(x_{i}, t_{i}\right)\right\}_{i=1}^{\infty}$ is dense on $\Phi$. Further, we suppose that

$$
\Psi_{i}(x, t)=\mathcal{L}^{*} \varphi_{i}(x, t),
$$

where $\mathcal{L}^{*}$ is the adjoint operator of $\mathcal{L}$.

In the following theorem, the main characteristics of the above concepts are summarized.

Theorem 2.2. If $\left\{\left(x_{i}, t_{i}\right)\right\}_{i=1}^{\infty}$ is dense on $\Phi$, then $\left\{\Psi_{i}(x, t)\right\}_{i=1}^{\infty}$ is a complete system of ${ }^{0} \omega_{2}^{(4,2)}(\Phi)$.

Proof 2.2. See [6].

\section{Numerical solution method for the Dym equation}

In this section, we provide two numerical methods for the solution of the Dym equation. The first of these will not employ orthogonalization, while the second will. The first method is therefore less computationally demanding (resulting in faster computation time), while the second method is more useful for convergence analysis (which is considered in the next section). We shall outline both methods here. Since the methods only differ based on the orthogonality of the base functions used in their respective approximations, either method can be used to solve the Dym equation. 


\subsection{Solution approach for Eq. (7) without orthogonalization}

In this section, we propose an iterative algorithm for solving Eq. (7), using the reproducing kernel space. We avoid having to use the Gram-Schmidt orthogonalization process in this method. One benefit to this is that the computational time can be lowered. The solution method of (7) is given in the following theorem.

Theorem 3.1. Let $\left\{\left(x_{i}, t_{i}\right)\right\}_{i=1}^{\infty}$ be a dense set on $\Phi$. If Equation (7) has a unique solution, then the solution satisfies the form

$$
\vartheta(x, t)=\sum_{i=1}^{\infty} \alpha_{i} \Psi_{i}(x, t),
$$

where the coefficients $\alpha_{i}$ are determined by solving the semi-infinite system of linear equations $\mathcal{B} \alpha=\mathcal{F}$, where

$$
\mathcal{B}=\left[\mathcal{L} \Psi_{i}\left(x_{j}, t_{j}\right)\right], \quad i, j=1,2, \ldots, \quad \alpha=\left[\alpha_{1}, \alpha_{2}, \ldots\right]^{T},
$$

and

$$
\mathcal{F}=\left[\mathcal{F}\left(x_{1}, t_{1}, \vartheta\left(x_{1}, t_{1}\right), \partial_{x x x} \vartheta\left(x_{1}, t_{1}\right)\right), \mathcal{F}\left(x_{2}, t_{2}, \vartheta\left(x_{2}, t_{2}\right), \partial_{x x x} \vartheta\left(x_{2}, t_{2}\right)\right), \ldots\right]^{T} .
$$

Proof 3.1. By Theorem 2.2, the solution can be represented as (9). Since

$$
\begin{aligned}
\left\langle\Psi_{j}(x, t), \Psi_{i}(x, t)\right\rangle_{\omega_{\omega_{2}^{(4,2)}}} & =\left\langle\mathcal{L}^{*} \varphi_{j}(x, t), \Psi_{i}(x, t)\right\rangle_{\omega_{\omega_{2}^{(4,2)}}} \\
& =\left\langle\varphi_{j}(x, t), \mathcal{L} \Psi_{i}(x, t)\right\rangle_{{ }_{0} \omega_{2}^{(1,1)}} \\
& =\mathcal{L} \Psi_{i}\left(x_{j}, t_{j}\right)
\end{aligned}
$$

and

$$
\begin{aligned}
\left\langle\vartheta(x, t), \Psi_{j}(x, t)\right\rangle_{\omega_{\omega_{2}}^{(4,2)}} & =\left\langle\vartheta(x, t), \mathcal{L}^{*} \varphi_{j}(x, t)\right\rangle_{0_{\omega_{2}^{(4,2)}}} \\
& =\left\langle\mathcal{L} \vartheta(x, t), \varphi_{j}(x, t)\right\rangle_{\omega_{2}^{(1,1)}} \\
& =\mathcal{F}\left(x_{j}, t_{j}, \vartheta\left(x_{j}, t_{j}\right), \partial_{x x x} \vartheta\left(x_{j}, t_{j}\right)\right) .
\end{aligned}
$$

According to the best approximation principle in Hilbert spaces [21], the coefficients $\alpha_{i}$ are determined by solving the semi-infinite system of linear equations $\mathcal{B} \alpha=\mathcal{F}$, and the proof is complete.

\subsection{Solution approach for Eq. (7) with orthogonalization}

Despite the fact that we can easily obtain solutions as discussed in the previous subsection, we note that by working with orthonormal basis elements, there will be some numerical advantages. For instance, there will be increased stability and lower computational complexity. Therefore, we shall derive an orthonormal basis system $\left\{\bar{\Psi}_{i}(x, t)\right\}_{i=1}^{\infty}$ of ${ }^{0} \omega_{2}^{(4,2)}$ from the Gram-Schmidt process applied to $\left\{\Psi_{i}(x, t)\right\}_{i=1}^{\infty}$. To do so, consider basis elements

$$
\bar{\Psi}_{i}(x, t)=\sum_{k=1}^{i} \rho_{i k} \Psi_{k}(x, t)
$$

where $\rho_{i k}$ are orthogonalization coefficients.

Regarding the solution method of Eq. (3), we have the following theorem.

Theorem 3.2. Let $\left\{\left(x_{i}, t_{i}\right)\right\}_{i=1}^{\infty}$ be dense on $\Phi$. If Equation (7) has a unique solution, then the solution satisfies the form

$$
\vartheta(x, t)=\sum_{i=1}^{\infty} \sum_{k=1}^{i} \rho_{i k} \mathcal{F}\left(x_{k}, t_{k}, \vartheta\left(x_{k}, t_{k}\right), \partial_{x x x} \vartheta\left(x_{k}, t_{k}\right)\right) \bar{\Psi}_{i}(x, t) .
$$


Proof 3.2. Assume that $\vartheta(x, t)$ is the solution of Eq. (7). By Theorem 2.2, $\vartheta(x, t)$ can be expanded in Fourier series as follows:

$$
\begin{aligned}
\vartheta(x, t) & =\sum_{i=1}^{\infty}\left\langle\vartheta(x, t), \bar{\Psi}_{i}(x, t)\right\rangle_{\omega_{2}(4,2)} \bar{\Psi}_{i}(x, t) \\
& =\sum_{i=1}^{\infty} \sum_{k=1}^{i} \rho_{i k}\left\langle\vartheta(x, t), \Psi_{k}(x, t)\right\rangle_{0} \omega_{2}^{(4,2)} \bar{\Psi}_{i}(x, t) \\
& =\sum_{i=1}^{\infty} \sum_{k=1}^{i} \rho_{i k}\left\langle\vartheta(x, t), \mathcal{L}^{*} \varphi_{k}(x, t)\right\rangle_{\omega_{2}^{(4,2)}} \bar{\Psi}_{i}(x, t) \\
& =\sum_{i=1}^{\infty} \sum_{k=1}^{i} \rho_{i k}\left\langle\mathcal{L} \vartheta(x, t), \varphi_{k}(x, t)\right\rangle_{\omega_{2}^{(1,1)}} \bar{\Psi}_{i}(x, t) \\
& =\sum_{i=1}^{\infty} \sum_{k=1}^{i} \rho_{i k} \mathcal{F}\left(x_{k}, t_{k}, \vartheta\left(x_{k}, t_{k}\right), \partial_{x x x} \vartheta\left(x_{k}, t_{k}\right)\right) \bar{\Psi}_{i}(x, t),
\end{aligned}
$$

and the proof is complete.

By truncating the series (9), we define the $m$-term approximation to $\vartheta(x, t)$ and obtain the approximate solution

$$
\vartheta_{m}(x, t)=\sum_{i=1}^{m} \kappa_{i} \bar{\Psi}_{i}(x, t)
$$

where

$$
\kappa_{i}=\sum_{k=1}^{i} \rho_{i k} \mathcal{F}\left(x_{k}, t_{k}, \vartheta\left(x_{k}, t_{k}\right), \partial_{x x x} \vartheta\left(x_{k}, t_{k}\right)\right)
$$

\section{Convergence analysis of the method}

In this section, convergence properties are discussed for the second algorithm. Since this method gives a rather convenient truncated representation for the approximate solution in terms of orthogonal base functions, we choose to work with this second numerical approach. However, we note that one could orthogonalize any base functions used in the approximate solution obtained using the first method, and hence the convergence results we obtain could be applied to the first method, as well.

Convergence of the sequence $\vartheta_{m}(x, t)$ of approximate solutions is provided in the following theorem.

Theorem 4.1. If $\vartheta(x, t) \in \Phi$, then there exists a constant $C>0$ such that

$$
\begin{gathered}
|\vartheta(x, t)| \leqslant C\|\vartheta(x, t)\|_{\omega_{\omega_{2}}^{(4,2)}}, \quad\left|\vartheta_{t}(x, t)\right| \leqslant C\|\vartheta(x, t)\|_{0} \omega_{2}^{(4,2)}, \\
\left|\vartheta_{x}(x, t)\right| \leqslant C\|\vartheta(x, t)\|_{0} \omega_{2}^{(4,2)}, \quad\left|\vartheta_{x x x x}(x, t)\right| \leqslant C\|\vartheta(x, t)\|_{0} \omega_{2}^{(4,2)}, \\
\left|\vartheta_{x x x t}(x, t)\right| \leqslant C\|\vartheta(x, t)\|_{\omega_{2}^{(4,2)}} .
\end{gathered}
$$

Proof 4.1. For any $(x, t) \in \Phi$, we have

$$
|\vartheta(x, t)|=\left|\left\langle\vartheta(\zeta, \eta), \Upsilon_{(x, t)}(\zeta, \eta)\right\rangle\right|_{0_{\omega_{2}^{(4,2)}}} \leqslant\|\vartheta(\zeta, \eta)\|_{\omega_{2}^{(4,2)}}\left\|\Upsilon_{(x, t)}(\zeta, \eta)\right\|_{\omega_{2}^{(4,2)}},
$$

therefore, there exists a constant $C_{1}>0$ such that

$$
|\vartheta(x, t)| \leqslant C_{1}\|\vartheta(\zeta, \eta)\|_{0} \omega_{2}^{(4,2)} .
$$


Also, we have

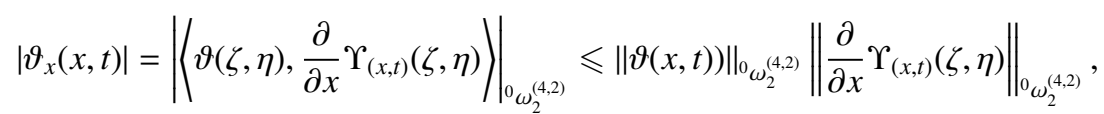

then there exists a constant $C_{2}>0$ such that

$$
\left|\vartheta_{x}(x, t)\right| \leqslant C_{2}\|\vartheta(\zeta, \eta)\|_{0} \omega_{2}^{(4,2)}
$$

In like manner, there exist constants $C_{3}, C_{4}, C_{5}>0$ such that

$$
\left|\vartheta_{t}(x, t)\right| \leqslant C_{3}\|\vartheta(x, t)\|_{0} \omega_{2}^{(4,2)}, \quad\left|\vartheta_{x x x t}(x, t)\right| \leqslant C_{4}\|\vartheta(x, t)\|_{0} \omega_{2}^{(4,2)},
$$

and

$$
\left|\vartheta_{x x x x}(x, t)\right| \leqslant C_{5}\|\vartheta(x, t)\|_{0} \omega_{2}^{(4,2)} .
$$

Let $C=\max \left\{C_{1}, C_{2}, C_{3}, C_{4}, C_{5}\right\}$, then the proof is completed.

As a direct consequence of the above theorem, we have the following result.

Theorem 4.2. If $\vartheta_{m}(x, t) \stackrel{\|\cdot\|_{\omega_{2}(4,2)}}{\longrightarrow} \bar{\vartheta}(x, t)$ as $m \rightarrow \infty,\left(x_{m}, t_{m}\right) \longrightarrow(x, t)$ as $m \rightarrow \infty,\left\|\vartheta_{m}(x, t)\right\|_{\omega_{2}^{(4,2)}}$ is bounded, and $\mathcal{F}\left(x, t, \vartheta(x, t), \partial_{x x} \vartheta(x, t)\right)$ is continuous, then

$$
\mathcal{F}\left(x_{m}, t_{m}, \vartheta_{m-1}\left(x_{m}, t_{m}\right), \partial_{x x x} \vartheta_{m-1}\left(x_{m}, t_{m}\right)\right) \longrightarrow \mathcal{F}\left(x, t, \bar{\vartheta}(x, t), \partial_{x x x} \bar{\vartheta}(x, t)\right)
$$

as $m \rightarrow \infty$.

Proof 4.2. Since

$$
\begin{aligned}
\left|\vartheta_{m-1}\left(x_{m}, t_{m}\right)-\bar{\vartheta}(x, t)\right| & \left.=\mid \vartheta_{m-1}\left(x_{m}, t_{m}\right)-\vartheta_{m-1}(x, t)+\vartheta_{m-1}(x, t)-\bar{\vartheta}(x, t)\right) \mid \\
& \leqslant\left|\partial_{x} \vartheta_{m-1}\right|\left|x_{m}-x\right|+\left|\partial_{t} \vartheta_{m-1}\right|\left|t_{m}-t\right|+\left|\vartheta_{m-1}(x, t)-\bar{\vartheta}(x, t)\right|
\end{aligned}
$$

and

$$
\begin{aligned}
& \left|\partial_{x x x} \vartheta_{m-1}\left(x_{m}, t_{m}\right)-\partial_{x x} \bar{\vartheta}(x, t)\right| \\
& \left.\quad=\mid \partial_{x x x} \vartheta_{m-1}\left(x_{m}, t_{m}\right)-\partial_{x x x} \vartheta_{m-1}(x, t)+\partial_{x x x} \vartheta_{m-1}(x, t)-\partial_{x x x} \bar{\vartheta}(x, t)\right) \mid \\
& \quad \leqslant\left|\partial_{x^{4}} \vartheta_{m-1}\right|\left|x_{m}-x\right|+\left|\partial_{x^{3} t} \vartheta_{m-1}\right|\left|t_{m}-t\right|+\left|\partial_{x x x} \vartheta_{m-1}(x, t)-\partial_{x x x} \bar{\vartheta}(x, t)\right|
\end{aligned}
$$

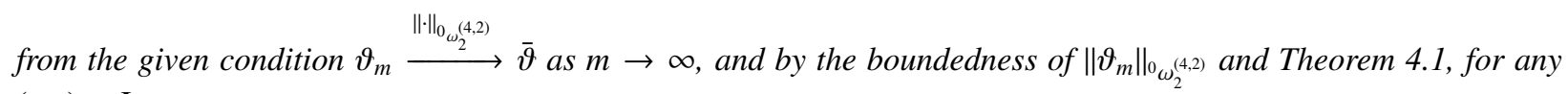
$(x, t) \in \Phi$, we get

$$
\left|\vartheta_{m-1}\left(x_{m}, t_{m}\right)-\bar{\vartheta}(x, t)\right| \rightarrow 0 \quad \text { and } \quad\left|\partial_{x x x} \vartheta_{m-1}\left(x_{m}, t_{m}\right)-\partial_{x x x} \bar{\vartheta}(x, t)\right| \rightarrow 0
$$

as $m \rightarrow \infty$. The continuation of $\mathcal{F}\left(x, t, \vartheta(x, t), \partial_{x x x} \vartheta(x, t)\right)$ implies that

$$
\mathcal{F}\left(x_{m}, t_{m}, \vartheta_{m-1}\left(x_{m}, t_{m}\right), \partial_{x x x} \vartheta_{m-1}\left(x_{m}, t_{m}\right)\right) \longrightarrow \mathcal{F}\left(x, t, \vartheta(x, t), \partial_{x x x} \vartheta(x, t)\right)
$$

as $m \rightarrow \infty$, which completes the proof.

Theorem 4.3. Suppose that $\left\|\vartheta_{m}(x, t)\right\|$ is bounded in (12). If $\left\{\left(x_{i}, t_{i}\right)\right\}_{i=1}^{\infty}$ is dense on $\Phi$, then the m-term approximate solution $\vartheta_{m}(x, t)$ derived from the above method converges to the exact solution $\vartheta(x, t)$ of Eq. (7), and

$$
\vartheta(x, t)=\sum_{i=1}^{\infty} \kappa_{i} \bar{\Psi}_{i}(x, t)
$$

where $\kappa_{i}$ is given by Eq. (13). 
Proof 4.3. First, we will prove the convergence of $\vartheta_{m}$. By Eq. (12), we infer that

$$
\vartheta_{m+1}(x, t)=\vartheta_{m}(x, t)+\kappa_{m+1} \bar{\Psi}_{m+1}(x, t) .
$$

From the orthogonality of $\left\{\bar{\Psi}_{i}(x, t)\right\}_{i=1}^{\infty}$, we conclude that

$$
\left\|\vartheta_{m+1}\right\|_{0} \omega_{2}^{(4,2)}=\left\|\vartheta_{m}\right\|_{0} \omega_{2}^{(4,2)}+\kappa_{m+1}^{2} .
$$

Hence it holds that $\left\|\vartheta_{m+1}\right\|_{0} \omega_{2}^{(4,2)} \geqslant\left\|\vartheta_{m}\right\|_{0} \omega_{2}^{(4,2)}$. By the boundedness of $\left\|\vartheta_{m}\right\|_{0} \omega_{2}^{(4,2),}\left\|\vartheta_{m}\right\|_{0} \omega_{2}^{(4,2)}$ is convergent and there exists a constant $c$ such that

$$
\sum_{i=1}^{\infty} \kappa_{i}^{2}=c .
$$

This implies that $\kappa_{i} \in l^{2}, \quad i=1,2, \ldots$ If $n>m$ and using the orthogonality of $\vartheta_{m+1}(x, t)-\vartheta_{m}(x, t)$, then we have

$$
\begin{aligned}
\left\|\vartheta_{n}(x, t)-\vartheta_{m}(x, t)\right\|_{0}^{2} \omega_{2}^{(4,2)} & \\
= & \left\|\vartheta_{n}(x, t)-\vartheta_{n-1}(x, t)+\vartheta_{n-2}(x, t)+\cdots+\vartheta_{m+1}(x, t)-\vartheta_{m}(x, t)\right\|_{0}^{2} \omega_{2}^{(4,2)} \\
& =\left\|\vartheta_{n}(x, t)-\vartheta_{n-1}(x, t)\right\|_{\omega_{2}^{(4,2)}}^{2}+\cdots+\left\|\vartheta_{m+1}(x, t)-\vartheta_{m}(x, t)\right\|_{\omega_{2}^{(4,2)}}^{2} \\
& =\sum_{i=m+1}^{n} \kappa_{i}^{2} \longrightarrow 0 \quad(m \longrightarrow \infty) .
\end{aligned}
$$

The completeness of ${ }^{0} \omega_{2}^{(4,2)}$ shows that $\vartheta_{m} \stackrel{\|\cdot\|_{\omega_{2}(4,2)}}{\longrightarrow} \bar{\vartheta} \quad$ as $\quad m \longrightarrow \infty$. Second, we will prove that $\bar{\vartheta}$ is the solution of Eq. (7). To this end, we conclude from (12) that

$$
\bar{\vartheta}(x, t)=\sum_{i=1}^{\infty} \kappa_{i} \bar{\Psi}_{i}(x, t) .
$$

Since

$$
\begin{aligned}
(\mathcal{L} \bar{\vartheta})\left(x_{m}, t_{m}\right) & =\sum_{i=1}^{\infty} \kappa_{i}\left\langle\mathcal{L} \bar{\Psi}_{i}, \varphi_{m}\right\rangle_{0} \omega_{2}^{(4,2)} \\
& =\sum_{i=1}^{\infty} \kappa_{i}\left\langle\bar{\Psi}_{i}, \Psi_{m}\right\rangle_{{ }_{\omega_{2}^{(4,2)}}}
\end{aligned}
$$

it follows that

$$
\begin{aligned}
\sum_{j=1}^{m} \rho_{m j}(\mathcal{L} \bar{\vartheta})\left(x_{m}, t_{m}\right) & =\sum_{i=1}^{\infty} \kappa_{i}\left\langle\bar{\Psi}_{i}, \sum_{j=1}^{m} \rho_{m j} \Psi_{j}\right\rangle_{{ }^{0} \omega_{2}^{(4,2)}} \\
& =\sum_{i=1}^{\infty} \kappa_{i}\left\langle\bar{\Psi}_{i}, \bar{\Psi}_{m}\right\rangle_{\omega_{2}^{(4,2)}}=\kappa_{m} .
\end{aligned}
$$

If $m=1$, then

$$
(\mathcal{L} \bar{\vartheta})\left(x_{1}, t_{1}\right)=\mathcal{F}\left(x_{1}, t_{1}, \vartheta_{0}\left(x_{1}, t_{1}\right), \partial_{x x x} \vartheta_{0}\left(x_{1}, t_{1}\right)\right)
$$

Also, for $m=2$, we have

$$
\begin{aligned}
\rho_{21}(\mathcal{L} \bar{\vartheta})\left(x_{1}, t_{1}\right)+\rho_{22}(\mathcal{L} \bar{\vartheta})\left(x_{2}, t_{2}\right)= & \rho_{21} \mathcal{F}\left(x_{1}, t_{1}, \vartheta_{0}\left(x_{1}, t_{1}\right), \partial_{x x x} \vartheta_{0}\left(x_{1}, t_{1}\right)\right) \\
& +\rho_{22} \mathcal{F}\left(x_{2}, t_{2}, \vartheta_{0}\left(x_{2}, t_{2}\right), \partial_{x x x} \vartheta_{0}\left(x_{2}, t_{2}\right)\right)
\end{aligned}
$$


It is clear that

$$
(\mathcal{L} \bar{\vartheta})\left(x_{2}, t_{2}\right)=\mathcal{F}\left(x_{2}, t_{2}, \vartheta_{0}\left(x_{2}, t_{2}\right), \partial_{x x x} \vartheta_{0}\left(x_{2}, t_{2}\right)\right) .
$$

On the whole, by induction, we conclude

$$
(\mathcal{L} \bar{\vartheta})\left(x_{j}, t_{j}\right)=\mathcal{F}\left(x_{j}, t_{j}, \vartheta_{j-1}\left(x_{j}, t_{j}\right), \partial_{x x x} \vartheta_{j-1}\left(x_{j}, t_{j}\right)\right) .
$$

For any $(\mu, \varrho) \in \Phi$, there exists a subsequence $\left\{\left(x_{m_{j}}, t_{m_{j}}\right)\right\}_{j=1}^{\infty}$ converging to $z$ since $\left\{\left(x_{i}, t_{i}\right)\right\}_{i=1}^{\infty}$ is dense in $\Phi$. Thus, by the convergence of $\vartheta_{m}$ and Theorem 4.2 , we conclude that

$$
(\mathcal{L} \bar{\vartheta})(\mu, \varrho)=\mathcal{F}\left(\mu, \varrho, \bar{\vartheta}(\mu, \varrho), \partial_{x x x} \bar{\vartheta}(\mu, \varrho)\right)
$$

That is, $\bar{\vartheta}(x, t)$ is the solution of Eq. (7) and

$$
\vartheta(x, t)=\sum_{i=1}^{\infty} \kappa_{i} \bar{\Psi}_{i}(x, t) .
$$

The proof is complete.

Theorem 4.4. Assume $\vartheta(x, t)$ is the solution of $E q .(7)$ and $\tau_{m}(x, t)$ is the approximate error between $\vartheta_{m}(x, t)$ and $\vartheta(x, t)$. Then the error $\tau_{m}(x, t)$ is monotone decreasing in the sense of $\|\cdot\|_{0} \omega_{2}^{(4,2)}$.

Proof 4.4. From (14) and according to the proof of Theorem 4.3, it follows that

$$
\left\|\tau_{m}\right\|_{\omega_{2}^{(4,2)}}^{2}=\left\|\sum_{i=m+1}^{\infty} \kappa_{i} \bar{\Psi}_{i}(x, t)\right\|_{0 \omega_{2}^{(4,2)}}^{2}=\sum_{i=m+1}^{\infty} \kappa_{i}^{2} .
$$

Eq. (21) shows that the error $\tau_{m}$ is monotone decreasing in the sense of $\|\cdot\|_{0} \omega_{2}^{(4,2)}$.

\section{Numerical experiments}

To verify applicability and accuracy of the proposed numerical method outlined in Section 3, we pick specific initial conditions in order to perform several numerical experiments. For a sufficient number of iterations, the results will converge for both methods, as outlined in Section 3. In order to same on computational time, we employ the first method. We find that taking $n=20$ is sufficient to give solutions with low error.

In general, there will not be exact solutions to the Dym equation for arbitrary initial conditions. In order to ascertain how accurate the numerical solutions are, we shall therefore consider the residual error obtained from plugging the approximate solution back into the Dym equation. We shall then determine the approximate solutions for various forms of the initial data $u(x, 0)=f(x)$, and use the relevant residual error to determine how useful the approximate solutions really are.

Let $E(x, t)$ denote the residual error at the points $(x, t) \in \Phi$. In Tables $1,2,3,4$, and 5, we provide $E(x, t)$ and the values of the approximate solution of $u_{n}(x, t)$ for arbitrary initial conditions $u(x, 0)=f(x)$. For the initial data used in Tables 1-(5), we give corresponding plots of the solutions in Figures 1-5, respectively. For many of the cases, the most rapid change in the solutions will occur for rather small time, with the solutions varying more slowly at time tends to unity.

\section{Conclusions}

In this paper we have considered two numerical solution approaches for the Dym initial value problem using the reproducing kernel Hilbert space method. For either approach given in Section 3, the solution is represented in the form of a series contained in the reproducing kernel space, and a truncated approximate solution is obtained. The two approaches differ in that the first does not require orthogonalization of the base functions (thereby saving on computational time), while the second approach employs and orthogonalization procedure so that the base functions 
are mutually orthogonal. Despite the increased computational time, the second approach is theoretically useful, as for instance it allows for a more straightforward convergence analysis. This convergence analysis was given in Section 4. Therefore, there are benefits to both approaches.

After developing the reproducing kernel Hilbert space method for the numerical solution of the Dym equation, we present several numerical experiments in Section 5, in order to demonstrate the utility of the method. For computational efficiency, we use the first method (with non-orthogonal base functions), noting that the convergence properties of this method are similar to those for the method which uses orthogonal base functions. Therefore, the theory predicts that solutions should converge to the true solution. Indeed, we see from the numerical solutions that absolute and residual errors are rather small after relatively few terms in the approximation expansion are used, suggesting that these approximate numerical solutions do, indeed, converge.

In summary, using residual errors as our metric, we found that that solving the Dym initial value problem using the reproducing kernel Hilbert space method resulted in fairly accurate solutions. Additionally, we showed theoretically that the method does give solutions which converge, and that the results were fairly straightforward and computationally efficient. Therefore, the results suggest that solving the Dym initial value problem in reproducing kernel space is a useful approach for obtaining accurate and efficient numerical solutions.

[1] S. Abbasbandy, R.A. Van Gorder, P. Bakhtiari, Reproducing kernel method for the numerical solution of the BrinkmanForchheimer momentum equation, J. Comput. Appl. Math., 311 (2017) 262-271.

[2] N. Aronszajn, The theory of reproducing kernels and their applications, Cambridge Philosophy Society Proceedings, 39 (1943) $133-153$.

[3] N. Aronszajn, Theory of reproducing kernels, Transactions of the American Mathematical Society, 68 no.3 (1950) $337-404$.

[4] S. Bergman, Uber Kurvenintegale von Funktionen zweier komplexen Veranderlichen, die Differentialgleichungen befriedigen, Math. Z., 32 (1930) 386-406.

[5] S. Bergman, The approximation of functions satisfying a linear partial differential equation, Duke Math. J., 6 (1940) 537-561.

[6] M.G. Cui, Y.Z. Lin, Nonlinear Numerical Analysis in Reproducing Kernel Space, Nova Science Pub. Inc., Hauppauge, 2009.

[7] M.G. Cui, F.Z. Geng, Solving singular two-point boundary value problem in reproducing kernel space, J. Comput. Appl. Math., 205 (2007) $6-15$.

[8] M.G. Cui, F.Z. Geng, A computational method for solving third-order singularly perturbed boundary-value problems, Appl. Math. Comput., 198 (2008) 896-903.

[9] L.A. Dmitrieva, The higher-times approach to multisoliton solutions of the Harry Dym equation, Journal of Physics A: Mathematical and General 26 (1993) 6005.

[10] F.Z. Geng, M.G. Cui, Solving singular nonlinear two-point boundary value problems in the reproducing kernel space, J. Korean Math. Soc., 45 (2008) 631-644.

[11] F. Z. Geng, S. P. Qian, and S. Li, A numerical method for singularly perturbed turning point problems with an interior layer, J. Comput. Appl. Math., 255 (2014) 97-105.

[12] F. Gesztesy, K. Unterkofler, Isospectral deformations for Sturm-Liouville and Dirac-type operators and associated nonlinear evolution equations, Reports on Mathematical Physics, 31 (1992) 113-137.

[13] W. Hereman, P.P. Banerjee, M.R. Chatterjee, Derivation and implicit solution of the Harry Dym equation and its connections with the Korteweg-de Vries equation, Journal of Physics A: Mathematical and General, 22 (1989) 241.

[14] L.P. Kadanoff, Exact solutions for the Saffman-Taylor problem with surface tension, Physical Review Letters, 65 (1990) $2986-2988$.

[15] M.D. Kruskal, Nonlinear wave equations, in: J. Moser (Ed.), Dynamical Systems, Theory and Applications, Lecture Notes in Physics, vol. 38, Springer, 1975.

[16] X.Y. Li, B.Y. Wu, Error estimation for the reproducing kernel method to solve linear boundary value problems, J. Comput. Appl. Math., 243 (2013) $10-15$

[17] X.Y. Li, B.Y. Wu, A novel method for nonlinear singular fourth order four-point boundary value problems, Comput. Math. Appl., 62 (2011) 27-31.

[18] X.Y. Li, B.Y. Wu, A continuous method for nonlocal functional differential equations with delayed or advanced arguments, J. Math. Anal. Appl., 409 (2014) 485-493.

[19] K. Mallory, R.A. Van Gorder, Method for constructing analytical solutions to the Dym initial value problem, Appl. Math. Comput., 226 (2014) 67-82.

[20] R. Mokhtari, Exact solutions of the Harry-Dym equation, Communications in Theoretical Physics 55 (2011) 204208.

[21] M. Mohammadi, R. Mokhtari, A reproducing kernel method for solving a class of nonlinear system of PDEs, Math. Model. Anal., 19 (2014) 180-198.

[22] D.P. Novikov, Algebraic-geometric solutions of the Harry Dym equation, Siberian Mathematical Journal, 40 (1999) 136-140.

[23] C. Rogers, The Harry Dym equation in $2+1$ dimensions: a reciprocal link with the KadomtsevPetviashvili equation, Physics Letters A, 120 (1987) 15-18

[24] Y.L. Wang, L. Chao, Using reproducing kernel for solving a class of partial differential equation with variable-coefficients, Appl. Math. Mech. Engl. Ed., 29(1) (2008) 129-137.

[25] Y.L. Wang, X.J. Cao, X.N. Li, A new method for solving singular fourth-order boundary value problems with mixed boundary conditions, Appl. Math. Comput., 217 (2011) 7385-7390.

[26] S. Zaremba, Sur le calcul numerique des founctions demandness dans le problems de dirichlet et le problems hydrodynamique, Bulletin International de I Academie des Sciences de Cracovie, (1908) 125-195. 


\begin{tabular}{lll}
\hline Nodes & $u_{20}(x, t)$ & $E(x, t)$ \\
\hline$(0.1,0.1)$ & 0.834516 & $9.27603 \times 10^{-15}$ \\
$(0.2,0.2)$ & 0.71014 & $7.76033 \times 10^{-15}$ \\
$(0.3,0.3)$ & 0.611929 & $5.999 \times 10^{-14}$ \\
$(0.4,0.4)$ & 0.531073 & $3: 31688 \times 10^{-15}$ \\
$(0.5,0.5)$ & 0.462576 & $3.04898 \times 10^{-15}$ \\
$(0.6,0.6)$ & 0.403326 & $1.75607 \times 10^{-15}$ \\
$(0.7,0.7)$ & 0.351246 & $1.36487 \times 10^{-16}$ \\
$(0.8,0.8)$ & 0.304875 & $3.13726 \times 10^{-16}$ \\
$(0.9,0.9)$ & 0.243682 & $1.77508 \times 10^{-15}$ \\
\hline
\end{tabular}

Table 1: Numerical results and residual error values for $f(x)=e^{-x}$.

\begin{tabular}{lll}
\hline Nodes & $u_{20}(x, t)$ & $E(x, t)$ \\
\hline$(0.1,0.1)$ & 0.554901 & $3.16854 \times 10^{-5}$ \\
$(0.2,0.2)$ & 0.368654 & $2.45444 \times 10^{-5}$ \\
$(0.3,0.3)$ & 0.262911 & $1.39747 \times 10^{-5}$ \\
$(0.4,0.4)$ & 0.174328 & $5.57413 \times 10^{-6}$ \\
$(0.5,0.5)$ & 0.156784 & $4.24041 \times 10^{-6}$ \\
$(0.6,0.6)$ & 0.134567 & $2.72376 \times 10^{-6}$ \\
$(0.7,0.7)$ & 0.125504 & $2.04479 \times 10^{-6}$ \\
$(0.8,0.8)$ & 0.126081 & $1.7752 \times 10^{-6}$ \\
$(0.9,0.9)$ & 0.133688 & $1.71678 \times 10^{-6}$ \\
\hline
\end{tabular}

Table 2: Numerical results and residual error values for $f(x)=e^{-2 x}$.

\begin{tabular}{lll}
\hline Nodes & $u_{20}(x, t)$ & $E(x, t)$ \\
\hline$(0.1,0.1)$ & 0.000400629 & $4.74069 \times 10^{-16}$ \\
$(0.2,0.2)$ & 0.00280876 & $1.69381 \times 10^{-16}$ \\
$(0.3,0.3)$ & 0.00913327 & $1.57419 \times 10^{-17}$ \\
$(0.4,0.4)$ & 0.0215015 & $3.3875 \times 10^{-15}$ \\
$(0.5,0.5)$ & 0.0419451 & $2.3992 \times 10^{-15}$ \\
$(0.6,0.6)$ & 0.072518 & $1.19903 \times 10^{-15}$ \\
$(0.7,0.7)$ & 0.115352 & $7.87004 \times 10^{-16}$ \\
$(0.8,0.8)$ & 0.172798 & $5.28943 \times 10^{-16}$ \\
$(0.9,0.9)$ & 0.247768 & $3.42105 \times 10^{-15}$
\end{tabular}

Table 3: Numerical results and residual error values for $f(x)=x^{3} / 3$. 


\begin{tabular}{lll}
\hline Nodes & $u_{20}(x, t)$ & $E(x, t)$ \\
\hline$(0.1,0.1)$ & 0.152488 & $8.61517 \times 10^{-13}$ \\
$(0.2,0.2)$ & 0.274706 & $1.08093 \times 10^{-12}$ \\
$(0.3,0.3)$ & 0.347502 & $2.86921 \times 10^{-11}$ \\
$(0.4,0.4)$ & 0.379517 & $3.13859 \times 10^{-11}$ \\
$(0.5,0.5)$ & 0.385233 & $3.96971 \times 10^{-12}$ \\
$(0.6,0.6)$ & 0.36965 & $1.42502 \times 10^{-10}$ \\
$(0.7,0.7)$ & 0.326568 & $5.47162 \times 10^{-10}$ \\
$(0.8,0.8)$ & 0.242359 & $1.00653 \times 10^{-11}$ \\
$(0.9,0.9)$ & 0.113282 & $4.20568 \times 10^{-11}$ \\
\hline
\end{tabular}

Table 4: Numerical results and residual error values for $f(x)=(1 / 2) \sin \pi x$.

\begin{tabular}{lll}
\hline Nodes & $u_{20}(x, t)$ & $E(x, t)$ \\
\hline$(0.1,0.1)$ & 0.100069 & $6.19097 \times 10^{-15}$ \\
$(0.2,0.2)$ & 0.196824 & $7.42826 \times 10^{-15}$ \\
$(0.3,0.3)$ & 0.288134 & $9.48858 \times 10^{-15}$ \\
$(0.4,0.4)$ & 0.372189 & $2.52549 \times 10^{-14}$ \\
$(0.5,0.5)$ & 0.44892 & $2.64826 \times 10^{-14}$ \\
$(0.6,0.6)$ & 0.519948 & $1.94272 \times 10^{-14}$ \\
$(0.7,0.7)$ & 0.587967 & $1.34886 \times 10^{-14}$ \\
$(0.8,0.8)$ & 0.656152 & $3.81416 \times 10^{-15}$ \\
$(0.9,0.9)$ & 0.727865 & $9.28292 \times 10^{-15}$ \\
\hline
\end{tabular}

Table 5: Numerical results and residual error values for $f(x)=\tanh x$.

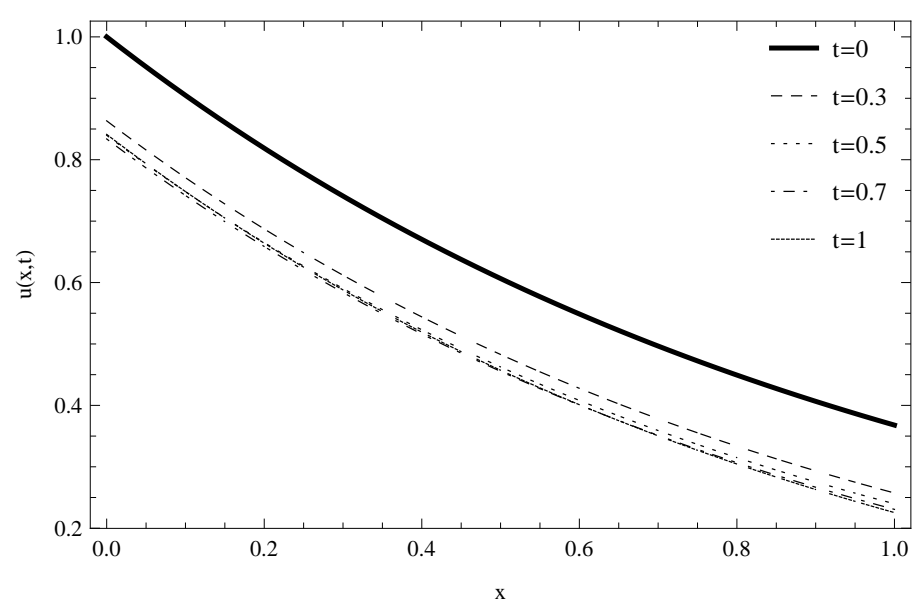

Figure 1: Approximate solutions of $u$ for 20-order RKS solution corresponding to initial data $u(x, 0)=f(x)=e^{-x}$. 


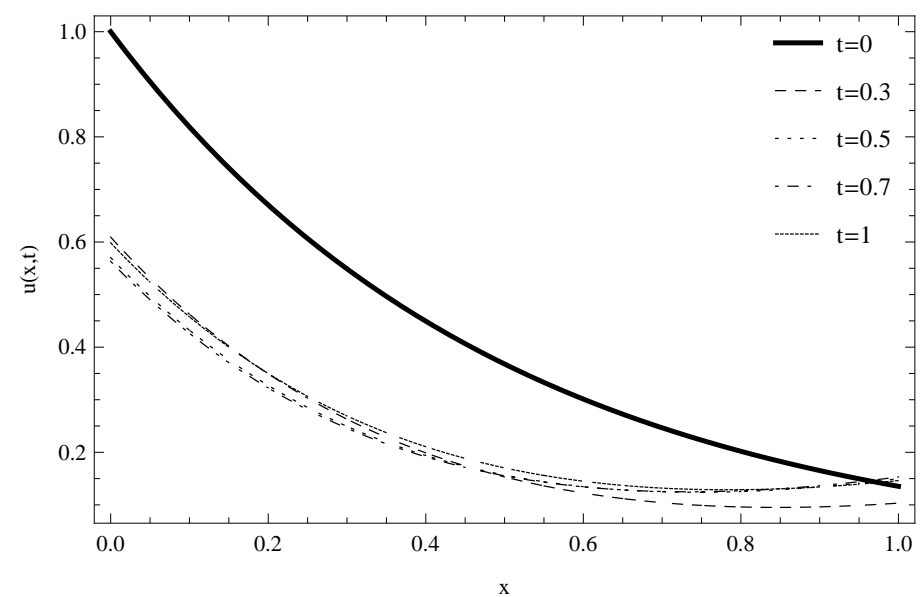

Figure 2: Approximate solutions of $u$ for 20-order RKS solution corresponding to initial data $u(x, 0)=f(x)=e^{-2 x}$.

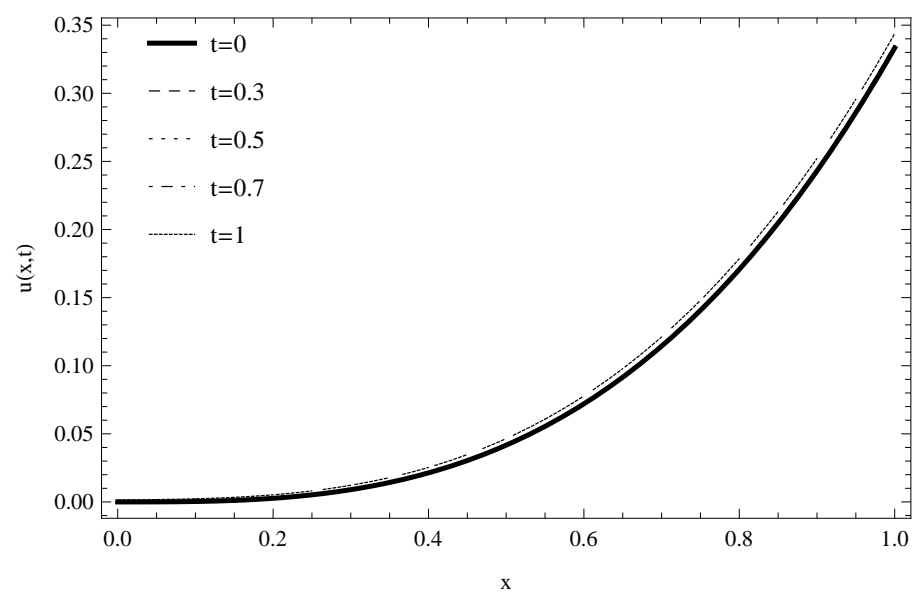

Figure 3: Approximate solutions of $u$ for 20-order RKS solution corresponding to initial data $u(x, 0)=f(x)=x^{3} / 3$.

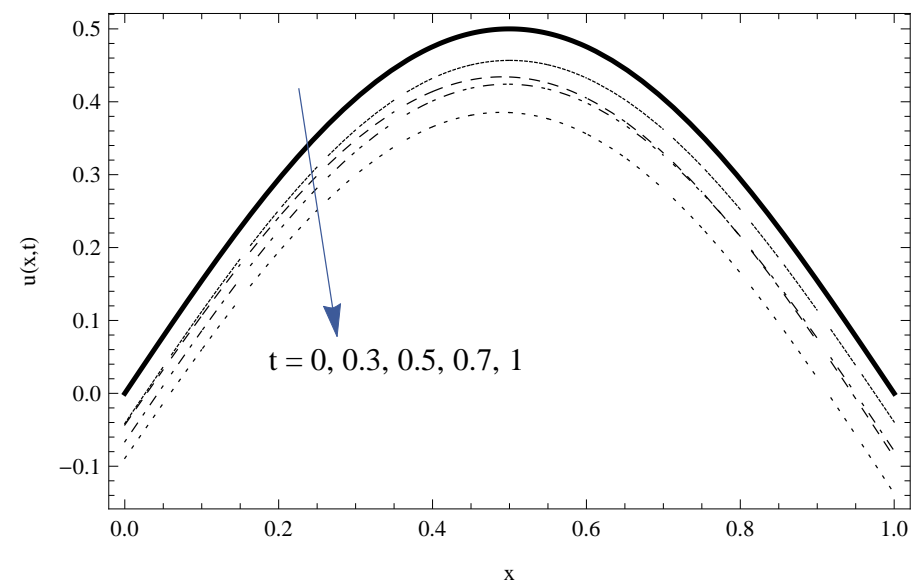

Figure 4: Approximate solutions of $u$ for 20-order RKS solution corresponding to initial data $u(x, 0)=f(x)=(1 / 2) \sin \pi x$. 


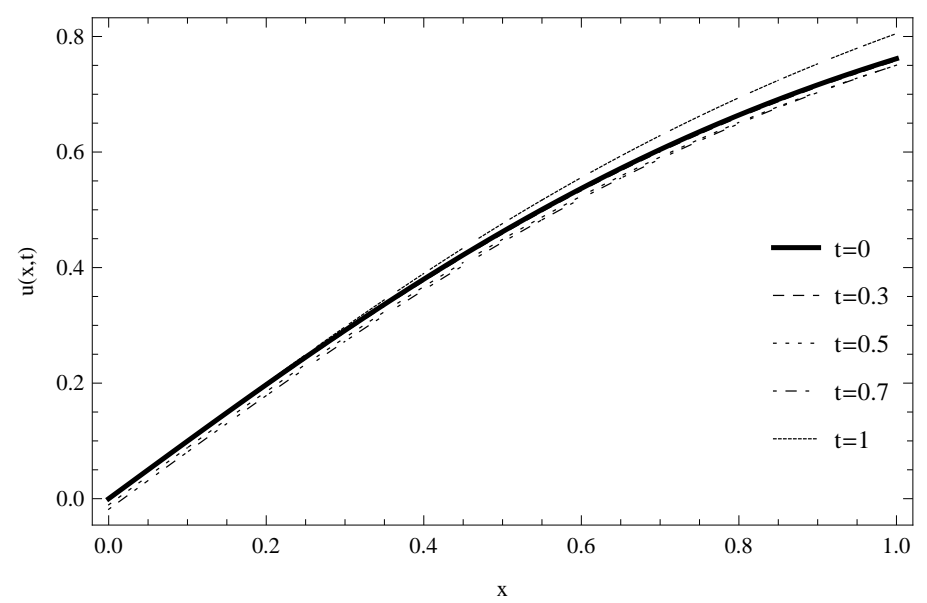

Figure 5: Approximate solutions of $u$ for 20-order RKS solution corresponding to initial data $u(x, 0)=f(x)=\tanh x$.

13 